\title{
PRIMITIVE GROUP RINGS AND NOETHERIAN RINGS OF QUOTIENTS
}

\author{
BY
}

CHRISTOPHER J. B. BROOKES AND KENNETH A. BROWN

\begin{abstract}
Let $k$ be a field, and let $G$ be a countable nilpotent group with centre $Z$. We show that the group algebra $k G$ is primitive if and only if $k$ is countable, $G$ is torsion free, and there exists an abelian subgroup $A$ of $G$, of infinite rank, with $A \cap Z=1$. Suppose now that $G$ is torsion free. Then $k G$ has a partial quotient ring $Q=k G(k Z)^{-1}$. The above characterisation of the primitivity of $k G$ is intimately connected with the question: When is $Q$ a Noetherian ring? We determine this for those groups $G$, as above, all of whose finite rank subgroups are finitely generated. In this case, $Q$ is Noetherian if and only if $G$ has no abelian subgroup $A$ of infinite rank with $A \cap Z=1$.
\end{abstract}

1. Introduction. This paper has two main themes. Both concern the group algebra $k G$ of a torsion free nilpotent group $G$. The first-the one we had in mind when we began our work -is the characterisation of those algebras $k G$ which are primitive, for $G$ countable. Let $Z$ denote the centre of $G$. The second theme is the determination of when the partial quotient ring $k G(k Z)^{-1}$ is Noetherian. (It is always simple.) It was a surprise to us that $k G(k Z)^{-1}$ frequently is Noetherian, and that this phenomenon is closely related to the primitivity of $k G$.

Let $\mathscr{P}$ denote the class of torsion free locally nilpotent groups whose finite rank subgroups are finitely generated. A subgroup $H$ of a group $G$ is dense if some positive power of every element of $G$ lies in $H$. Countable locally nilpotent groups contain dense $\mathscr{P}$-subgroups (Lemma 5.1(ii)). Our central result is

THEOREM D. Let $G$ be a countable hypercentral group with $G \neq 1$. Let $H$ be a dense

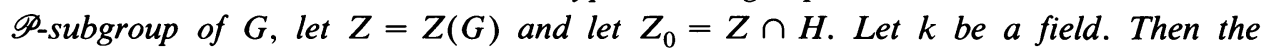
following are equivalent:

(a) $k G$ is primitive;

(b) $k$ is countable, $G$ is torsion-free and there exists an abelian subgroup $A$ of $G$, of infinite rank, with $A \cap Z=1$;

(c) $k H\left(k Z_{0}\right)^{-1}$ is not Noetherian, $G$ is torsion-free and $k$ is countable.

Most of the arguments used in proving Theorem $\mathrm{D}$ actually apply to a somewhat larger class of groups, namely the class of countable torsion-free hyperabelian locally nilpotent groups. So Theorem D is a refinement of Theorem C, a less precise result concerning the group algebras of groups in this class.

Received by the editors May 16, 1984.

1980 Mathematics Subject Classification. Primary 16A33, 16A27; Secondary 20C07, 16A20.

Kev words and phrases. Group ring, primitive ring, Noetherian ring, nilpotent group. 
The sufficiency of the conditions in Theorem $\mathrm{D}(\mathrm{b})$ for primitivity is an easy consequence of one of Zalesskii's intersection theorems, and was already observed in [3, Lemma 3.1]. Nor is it at all difficult to see that (a) implies (c): The argument is in two parts. First one observes that if $k G$ is primitive, then so is $k H$ (Lemma 7.4). Secondly, if $k H\left(k Z_{0}\right)^{-1}$ is Noetherian, then any cyclic $k Z_{0}$-torsion free $k H$-module $V$ is easily seen to be induced from a cyclic module $W$ for a subalgebra $k C$, where $C / Z_{0}$ is polycyclic, and one deduces from a stronger form [2] of P. Hall's generic flatness result [8, Lemma 4.1] that $W$ (and so $V$ ) cannot be irreducible.

The deepest part of Theorem D is thus the fact that (c) implies (b). This implication is part of our second main result:

TheOREM B. Let $R$ be a commutative domain and let $G$ be a torsion-free locally nilpotent hyperabelian $\mathscr{P}$-group. Let $B$ be a normal subgroup of $G$ with $G / B \in \mathscr{P}$, and let $Q G$ denote $R G(R B)^{-1}$. Then the following are equivalent:

(a) $Q G$ is Noetherian;

(b) the set of subgroups of $G$ having trivial intersection with $B$ satisfies the ascending chain condition;

(c) $G$ has no abelian subgroup $A$ of infinite rank with $B \cap A=1$.

Note that if $G$ is a hypercentral $\mathscr{P}$-group, then $G / Z(G) \in \mathscr{P}$ (Lemma 5.1(i)). To see that $k G(k B)^{-1}$ exists in the circumstances of Theorem $\mathrm{B}$, note that $k B$ is an Ore domain since it has these properties locally, $B$ being locally-(poly-(infinite cyclic)). Then [14, Lemma 2.6] shows that $k B-\{0\}$ is an Ore set in $k G$.

The free nilpotent groups $G_{c}$ of class $c(c \geqslant 2)$ on a countably infinite generating set provide instructive examples for Theorems B and D. Each $G_{c}$ is a $\mathscr{P}$-group. Every abelian subgroup having trivial intersection with the centre $Z$ of $G_{2}$ is cyclic; so, for any field $k, k G_{2}(k Z)^{-1}$ is Noetherian and $k G$ is not primitive. However, if $c \geqslant 3, G_{c}$ contains an abelian subgroup of infinite rank which meets the centre of $G_{c}$ in $\{1\}$, so $k G_{c}(k Z)^{-1}$ is not Noetherian, and $k G_{c}$ is primitive if and only if $k$ is countable. (In his Ph.D. thesis (Leeds University, 1984), J. Pettit also shows that $k G_{2}(k Z)^{-1}$ is Noetherian.)

The difficult part of Theorem B is the statement that (b) implies (a). The crux of the proof rests in showing that, given (b), if $M$ is a nonzero $Q G$-module, then $M$ contains a nonzero $k G$-module induced from a subalgebra $k D$, where $B \triangleleft D$ and $D / B$ is polycyclic. The construction depends on properties of modules over group algebras of finitely generated nilpotent groups, and in particular on a result on the "lifting of induction" (Theorem A) which may have other applications.

The results are arranged as follows. $\$ 2$ contains frequently used notation and terminology. $\S 3$ is a collection of module theoretic lemmas. Theorem A is proved in $\S 4$. A few group theoretic results are gathered together in $\S 5$. Theorem B is proved in $\S 6$, and Theorems C and D in $\S 7$.

ACKNowledgement. Some of the research described here was done at the University of Warwick during a symposium on Noetherian rings held there in 1981-82. We are grateful to the staff of the Mathematics Institute, University of Warwick, for their hospitality, and to the S.E.R.C. for its financial support. 
2. Notations and conventions. "Module" will always mean "unital right module". If $S$ is a ring and $M$ is an $S$-module, $k-\operatorname{dim}_{S}(M)$, or $k$-dim( $\left.M\right)$, denotes the Krull dimension of $M$ (if it exists) in the sense of Gabriel and Rentschler (see [6]); and $u$ - $\operatorname{dim}_{S}(M)$ or $u$-dim( $\left.M\right)$ denotes the uniform or Goldie dimension of $M$ [9, Theorem 10.4.4]. The annihilator of $M,\{s \in S: M s=0\}$, is denoted $\operatorname{Ann}_{S}(M)$ or $\operatorname{Ann}(M)$. The $S$-modules $M$ and $N$ are similar if their injective hulls are isomorphic. In other words, $M$ is similar to $N$ if there exist essential submodules $M^{\prime}$ of $M$ and $N^{\prime}$ of $N$ with $M^{\prime} \cong N^{\prime}$. A nonzero $S$-module is critical if it is finitely generated, has Krull dimension, and all its proper images have Krull dimension strictly less than that of $M$.

If $I$ is an ideal of the group ring $R G$, then $I^{+}$will denote the normal subgroup $\{g \in G: g-1 \in I\}$ of $G$. Let $G$ be polycyclic-by-finite and $R$ commutative Noetherian. A nonzero $R G$-module $M$ is called impervious if, for all subgroups $H$ of infinite index in $G$, there is no nonzero submodule of $M$ of the form $U \otimes_{R H} R G$ for an $R H$-module $U$.

The Hirsch number of the finitely generated nilpotent group $G$ will be denoted by $h(G)$. If $H$ is a subgroup of a locally nilpotent group $G$, the isolator of $H$ in $G$, $\left\{x \in G: x^{n} \in H\right.$ for some $\left.n \geqslant 1\right\}$, will be denoted is ${ }_{G}(H)$ or is $(H)$-it is a subgroup of $G$. We say that $H$ is isolated (in $G$ ) if is ${ }_{G}(H)=H$, and dense in $G$ if is $_{G}(H)=G$.

A commutative Noetherian domain $R$ is absolutely Hilbert if each prime ideal of $R$ is an intersection of maximal ideals, and all its field images are algebraic extensions of finite fields.

\section{Module theoretic lemmas.}

LEMMA 3.1. Let $S$ be a ring and $M$ an $S$-module which is a sum of uniform similar $S$-modules $W_{i}(i \in I)$. Suppose $M$ contains no $S$-module isomorphic to a submodule of a proper quotient of one of the $W_{i}$. Let $B$ be a uniform submodule of $M$. Then $B$ is similar to each of the $W_{i}$.

Proof. There is no harm in assuming that $B$ is finitely generated, so $M=W_{1}+$ $W_{2}+\cdots+W_{n}$, say. We construct an essential submodule $N=N_{1} \oplus \cdots \oplus N_{t}$ of $M$ with each $N_{i}$ similar to $W_{i}$. Put $N_{1}=W_{1}$ and suppose $N_{1} \oplus \cdots \oplus N_{i}$ has been defined and is essential in $V_{j}=W_{1}+\cdots+W_{j}$, where $1 \leqslant i \leqslant j<n$. If $N_{1} \oplus \cdots \oplus N_{i}$ is essential in $V_{j+1}=W_{1}+\cdots+W_{j+1}$, no more need be said. Otherwise, there exists a nonzero submodule $T$ of $V_{j+1}$ with $T \cap V_{j}=0$, so

$$
T=T / T \cap V_{j} \cong T+V_{j} / V_{j} \subseteq V_{j+1} / V_{j} \cong W_{j+1} / W_{j+1} \cap V_{j}
$$

If $W_{j+1} \cap V_{j} \neq 0$, then $T$ is an $S$-submodule of $M$ isomorphic to a submodule of a proper quotient of $W_{j+1}$, contradicting the hypothesis. Thus $W_{j+1} \cap V_{j}=0$ and we let $N_{i+1}$ be $W_{j+1}$. In this way $N$ can be constructed.

Now $N \cap B$ is nonzero and uniform, so one of the projection maps from $N \cap B$ to $N_{i}$ is a monomorphism. Thus $N \cap B$ can be embedded in $N_{i}$; since $N_{i}$ is similar to $W_{1}$, the proof is complete. 
The next lemma is an attempt to retrieve some aspects of Clifford's theorem (see [5, Theorem 11.1] for instance) for infinite groups and for modules which are not necessarily Artinian.

LEMMA 3.2. Let $R$ be a ring and $H$ a normal subgroup of the group $G$. Let $W$ be an $R H$-submodule of an $R G$-module and define $L=\{g \in G: W g$ is $R H$-similar to $W\}$.

(i) $L$ is a subgroup of $G$.

(ii) Suppose that $W$ is a uniform $R H$-submodule of an $R G$-module $M$, and that every nonzero submodule of WRL contains a uniform RH-module. Suppose there are no $R H$-submodules of $M$ isomorphic to a submodule of a proper quotient of $W$. Then $W R G=W R L \otimes_{R L} R G$.

Proof. (i) If $g \in L$ and $A \subseteq W, B \subseteq W g$ with $\psi: A \rightarrow B$ an $R H$-isomorphism, then $A g^{-1} \subseteq W g^{-1}$ and $B g^{-1} \subseteq W$ are isomorphic via the $R H$-map $\psi^{\prime}: a g^{-1} \mapsto$ $\psi(a) g^{-1}$. Suppose that $g, u \in L$, so there are essential $R H$-submodules $A \subseteq W g, B$ and $C$ in $W$, and $D \subseteq W u$, with $R H$-isomorphisms $\psi: A \rightarrow B$ and $\varphi: C \rightarrow D$. As above, there is an $R H$-isomorphism $\varphi^{\prime}$ between $C g \subseteq W g$ and $D g \subseteq W(u g)$. Now $A \cap C g$ is essential in $W g$. Thus $\varphi^{\prime} \psi$ defines an $R H$-isomorphism between the essential submodules $\psi^{-1}(A \cap C g)$ of $W$ and $\varphi^{\prime}(A \cap C g)$ of $W(u g)$.

(ii) Let $\left\{g_{i}: i \in I\right\}$ be a right transversal to $L$ in $G$. Suppose there exists an $R H$-module $B$ with

$$
0 \neq B \subseteq W R L \cap \sum_{\substack{i \in I \\ g_{i} \notin L .}} W R L g_{i} .
$$

By hypothesis we can choose $B$ finitely generated and uniform, in such a way that (rewriting part of the index set $I$ and with $g_{1} \in L$ ),

$$
0 \neq B \subseteq W R L \cap \sum_{i=2}^{n} W R L g_{i},
$$

with $n$ as small as possible. Consequently, as $R L$-modules,

$$
\sum_{i=2}^{n} W R L g_{i}=\sum_{i=2}^{n} \oplus R L g_{i} \text {. }
$$

The $R H$-projections $\pi_{i}: B \rightarrow W R L g_{i}(2 \leqslant i \leqslant n)$ are thus well defined, and $\cap_{i=2}^{n} \operatorname{ker} \pi_{i}=0$. The uniformity of $B$ forces $\operatorname{ker} \pi_{i}=0$ for some $i$; hence, for this $i, B$ is similar to a uniform $R H$-submodule of $W R L g_{i}$. Since $B \subseteq W R L, B$ is similar to $W$ by Lemma 3.1 and the supposition about sections of $W$. In view of Lemma 3.1 and the definition of $L$, no such $B$ can exist. Thus (ii) is proved.

Recall that a nonzero $R G$-module is impervious if it contains no nonzero submodule which is induced from a subgroup of infinite index in $G$.

LemMA 3.3. Let $H$ be a normal subgroup of a polycyclic-by-finite group $G$ with $G / H$ infinite cyclic. Let $R$ be a commutative Noetherian ring and let $W$ be an impervious $R G$-module. Then $W$ contains a nonzero $R G$-submodule $W_{1}$ such that

$$
u-\operatorname{dim}_{R H}\left(W_{1}\right)<\infty .
$$


Proof. Let $G=\langle H, x\rangle$ and let $U=u R H$ be a critical $R H$-submodule of $W$ of smallest Krull dimension. Since $W$ is impervious there exist $n \geqslant 1$ and $u^{\prime} \in U$ such that $U^{\prime}:=U+U x+\cdots+U x^{n-1}$ is a direct sum and

$$
0 \neq u^{\prime} \alpha_{n} x^{n}=\sum_{i=0}^{n-1} u^{\prime} \alpha_{i} x^{i} \in U x^{n} \cap U^{\prime},
$$

where $\alpha_{0}, \ldots, \alpha_{n} \in R H$. It is clearly enough to prove that $U^{\prime}$ is $R H$-essential in $U R G$.

To prove this, we argue by induction to show that, for all $m \geqslant n$ and $t \geqslant 0$,

$$
U^{\prime} \text { is essential in } \sum_{i=-t}^{m} U x^{i} \text {. }
$$

Fix $t \geqslant 0, r>n$ and suppose that (2) has been proved for the chosen $t$ and for $m<r$. Then by (1)

$$
0 \neq u^{\prime} \alpha_{n} x^{r}=\sum_{i=0}^{n-1} u^{\prime} \alpha_{i} x^{i+r-n} \in U x^{r} \cap\left(\sum_{i=0}^{r-1} U x^{i}\right) .
$$

Put $V_{r}=\sum_{i=0}^{r} U x^{i}$, and let $0 \neq T \subseteq V_{r}$ satisfy $T \cap U^{\prime}=0$. Then

$$
\begin{aligned}
T & =T / T \cap V_{r-1} \cong T+V_{r-1} / V_{r-1} \\
& \subseteq V_{r} / V_{r-1} \cong U x^{r} / U x^{r} \cap V_{r-1} .
\end{aligned}
$$

Since $U x^{r}$ is $R H$-critical, $k-\operatorname{dim}_{R H}(T)<k$ - $\operatorname{dim}_{R H}(U)$ by (4) and (3). This contradicts the choice of $U$, and so proves the induction step for $m$. Similar arguments deal with induction in $t$, and so (2) is proved. Thus the result follows.

LEMMA 3.4. Let $\sigma$ be an automorphism of $a$ ring $R$ and $S$ a subring of $R$ with $\sigma(S)=S$. Let $T=R\left[X, X^{-1} ; \sigma\right]$ and $T_{0}=S\left[X, X^{-1} ; \sigma\right]$. Let $V$ be an $R$-module with $u$-dim ${ }_{S}(V)=n<\infty$. Then $u$ - $\operatorname{dim}_{T_{0}}\left(V \otimes_{R} T\right)=n$.

Proof. The argument used in [13, Proposition 2.4] can easily be adapted to show that if $v S$ is a cyclic uniform $S$-submodule of $V$, then $v S\left[X, X^{-1} ; \sigma\right]$ is a uniform $S\left[X, X^{-1} ; \sigma\right]$-module. It follows at once that $u$-dim $\operatorname{dim}_{S}(V)=u$-dim $T_{0}\left(V T_{0}\right) . \operatorname{But} V$ is an $R$-module, so $V T_{0}=V T$.

LEMMA 3.5. Let $R$ be a commutative Noetherian ring and let $H$ be an isolated normal subgroup of the finitely generated nilpotent group $G$. Let $W$ be an $R H$-module. Then every nonzero $R G$-submodule $V$ of $W \otimes_{R H} R G$ contains a nonzero submodule induced from $R H$.

Proof. Clearly we may assume that $W$ (and so $V$ ) are finitely generated. Thus there exist a finitely generated subring $S$ of $R$, an $S H$-submodule $W_{1}$ of $W$, and an $S G$-submodule $V_{1}$ of $V$ such that $V=V_{1} \otimes_{S G} R G$ and $W=W_{1} \otimes_{S H} R H$, with $V_{1} \subseteq W_{1} \otimes_{S H} S G$. Note that $S$ is absolutely Hilbert. Also, any conclusions about the existence of an induced module pass immediately from $S G$ to $R G$. So we may assume that $R$ is absolutely Hilbert.

Let $Y=Y_{1} \oplus Y_{2} \oplus \cdots \oplus Y_{t}$ be an essential submodule of $W$, with each $Y_{i}$ critical. Let $V$ be a nonzero $R G$-submodule of $W \otimes R G$. Then $V \cap Y R G \neq 0$ by 
Lemma 3.4, so there is no loss in assuming $V$ is a uniform submodule of $Y \otimes R G$. Then one of the projection maps $\pi_{i}: V \rightarrow Y_{i} \otimes R G$ is a monomorphism, so we may replace $W$ by $Y_{i}$ and assume that $W$ is critical.

Let $k-\operatorname{dim}_{R H}(W)=s$. By $h(G / H)$ applications of [12, Lemma 10], WRG is critical of dimension $r=s+h(G / H)$. Let $V$ be a nonzero submodule of $W R G$ and let $V_{1}$ be a critical $R H$-submodule of $V$. Since $V_{1}$ lies in a finite direct sum of conjugates of $W$ and projects nontrivially into one of the summands, $k$-dim $\operatorname{dim}_{R}\left(V_{1}\right)$ $=s$. Therefore, applying [12, Lemma 10] $h(G / H)$ times, we deduce that

$$
V_{1} R G=V_{1} \otimes_{R H} R G \text {. }
$$

Lemma 3.6. Let $R$ be a commutative Noetherian ring and $G$ a finitely generated nilpotent group with isolated normal subgroup $H$. Let $W_{1}$ and $W_{2}$ be finitely generated $R H$-modules such that no nonzero submodule of any conjugate $W_{1} g$ (where $g \in G$ ) is isomorphic to a submodule of a proper quotient of $W_{2}$. Then no nonzero submodule of $W_{1} \otimes_{R H} R G$ is isomorphic to a submodule of a proper quotient of $W_{2} \otimes_{R H} R G$.

Proof. Since $H$ is isolated in $G$ there is a chain $H=H_{1} \subseteq \cdots \subseteq H_{r}=G$ of normal subgroups of $G$ with each $H_{i+1} / H_{i}$ infinite cyclic. The proof is by an inductive argument along the chain. As our inductive hypothesis we suppose that no nonzero submodule of $W_{1} g \otimes R H_{i}$, for any $g \in G$, is isomorphic to a submodule of a proper quotient of $W_{2} \otimes R H_{i}$.

Let $V$ be a nonzero $R H_{i+1}$-submodule of $W_{1} g \otimes R H_{i+1}$. By Lemma 3.5, $V$ contains a nonzero submodule induced from $R H$. But any nonzero $R H$-submodule of $W_{1} g \otimes R H_{i+1}$ has a nonzero submodule isomorphic to $W^{\prime} g h_{i+1}$, for some $h_{i+1} \in H_{i+1}$ and some $R H$-submodule $W^{\prime}$ of $W_{1}$. So $V$ has a nonzero submodule isomorphic to $W^{\prime} g h_{i+1} \otimes R H_{i+1}$; that is, to $W^{\prime} g \otimes R H_{i+1}$. If $V$ were isomorphic to a submodule of a proper quotient of $W_{2} \otimes R H_{i+1}$, then $W^{\prime} g \otimes R H_{i+1}$ would be too.

Now let $H_{i+1}=\left\langle H_{i}, x\right\rangle$ and $U=W_{2} \otimes R H_{i}$. We consider a proper quotient of $W_{2} \otimes R H_{i+1}=U \otimes R H_{i+1}$. Denote images in this quotient by an overbar. For some $n>0$ and $u_{0}, \ldots, u_{n} \in U$,

$$
\bar{u}_{0}+\cdots+\bar{u}_{n} x^{n}=\overline{0}, \quad u_{0}, u_{n} \neq 0 .
$$

Suppose an $R H_{i}$-submodule $\bar{U}^{\prime}$ of $\overline{U R H}_{i+1}$ is isomorphic to $W g h_{i+1} \otimes R H_{i}$. We claim that

$$
\bar{U}^{\prime} \cap \sum_{j=0}^{n-1} \bar{U} x^{j} \neq 0 .
$$

To prove (6), suppose

$$
\bar{U}^{\prime} \cap \sum_{j=-t}^{s} \bar{U} x^{j} \neq 0,
$$

with $s \geqslant n-1$ and $t \geqslant 0$. Then (5) implies that, if $s>n-1$,

$$
\sum_{j=-t}^{s} \bar{U} x^{j} / \sum_{j=-t}^{s-1} \bar{U} x^{j}
$$


is isomorphic to a proper quotient of $U x^{s}$. If $\bar{U}^{\prime} \cap \sum_{j=-t}^{s-1} \bar{U} x^{j}=0$, then $\bar{U}^{\prime}$ is isomorphic to a submodule of a proper quotient of $\bar{U} x^{s}$. But $\bar{U}^{\prime} \cong W^{\prime} g h_{i+1} \otimes R H_{i}$, and so $W^{\prime} g h_{i+1} x^{-s} \otimes R H_{i}$ is isomorphic to a submodule of a proper quotient of $\bar{U}$, and hence of $U$. This contradicts the inductive hypothesis. Similarly we can deduce that if $t>0$, then $\bar{U}^{\prime} \cap \sum_{j=-t+1}^{s} \bar{U} x^{j} \neq 0$. This proves (6).

Now $\sum_{j=0}^{n-1} \bar{U} x^{j}$ is a finitely generated $R H_{i}$-module, and is thus of finite uniform dimension. Also, $W^{\prime} g \otimes R H_{i+1}$ is the direct sum of the $R H_{i}$-submodules $W^{\prime} g x^{j} \otimes$ $R H_{i}$ for $-\infty<j<\infty$. Thus, in view of (6), $W^{\prime} g \otimes R H_{i+1}$ cannot be embedded in $\overline{U R H}_{i+1}$, an arbitrary proper quotient of $W_{2} \otimes R H_{i+1}$. Hence there is no nonzero submodule of $W_{1} g \otimes R H_{i+1}$ which can be embedded in a proper quotient of $W_{2} \otimes R H_{i+1}$, and the inductive step of the proof is established.

4. A control theorem for certain modules. This section is devoted to the proof of the following result, which is needed for the proof of Theorem B.

THEOREM A. Let $R$ be a commutative Noetherian domain, and $G$ a group. Let $H$ be a finitely generated nilpotent normal subgroup of $G$ and $K$ an isolated subgroup of $H$. Let $W$ be an $R K$-submodule of an RG-module $M$. Suppose that

(i) $W$ is uniform and impervious;

(ii) there exist no nonzero $R H$-submodules of $M$ isomorphic to a submodule of a proper quotient of $W R H$;

(iii) $W R H=W \otimes_{R K} R H$.

Put $N=N_{G}(K)$. Then $W R G=W R N \otimes_{R N} R G$.

Proof. Step 1. The induction set-up. For $i \geqslant 1$ we define subgroups $K_{i}$ of $H$ as follows. Put $K_{1}=H$ and for $i \geqslant 2$ set $K_{i}=$ is $_{K_{i-1}}\left(K\left(K_{i-1}\right)^{\prime}\right)$. An easy induction shows that, for $i \geqslant 1$,

$$
K_{i} \subseteq \operatorname{is}_{H}(\underbrace{[H, \ldots, H]}_{i}) .
$$

Since $H$ is nilpotent,

$$
\underbrace{[H, \ldots, H]}_{s}=1
$$

for some $s$, so that $K_{s}=$ is $_{H}(K)=K$.

We shall also define subgroups $L_{i}$ of $G$ for $i \geqslant 0$, satisfying

$$
K_{i+1} \triangleleft L_{i} .
$$

Put $L_{0}=G$, so that $(1 ; 0)$ is certainly true. The definition of the $L_{i}$ 's is an inductive one. After each $L_{i}$ is defined we must establish that $(1 ; i)$ is true; this constitutes Step 2 of the proof. Assuming $(1 ; i)$, we can define

$$
L_{i+1}=\left\{g \in L_{i}: W R K_{i+1} g \text { is } R K_{i+1} \text {-similar to } W R K_{i+1}\right\} \text {. }
$$

By $(1 ; i)$, this definition makes sense, and $L_{i+1}$ is a subgroup of $L_{i}$ by Lemma 3.2(i). We aim to apply part (ii) of that lemma with $K_{i+1}$ in the role of $H$ and $L_{i}$ in that of $G$, so we must show that $W R K_{i+1}$ is uniform. Since $K$ is isolated in $H$, there is a 
subnormal series between $K$ and $K_{i+1}$ with infinite cyclic factors. Thus $h\left(K_{i+1}\right)-$ $h(K)$ applications of Lemma 3.4 establish that $W R K_{i+1}$ is uniform. Then Lemma 3.2 (ii) yields

$$
W R L_{i}=W R L_{i+1} \otimes_{R L_{i, 1}} R L_{i} .
$$

Because $K_{s}=K,(1 ; s-1)$ shows that $L_{s-1}$ normalizes $K$. So $(2 ; s-2)$ and its predecessors yield the theorem.

It remains to prove $(1 ; i)$ after $L_{i}$ has been defined (so that $L_{i+1}$ can be defined).

Step 2. Proof of $(1 ; i)$. It is clear from the definition of $L_{i}$ that $K_{i} \subseteq L_{i}$. But $K_{i+1} \subseteq K_{i}$ and so $K_{i+1} \subseteq L_{i}$. Consider an isolated subgroup $D$ of $K_{i}$ with $K_{i}^{\prime} \subseteq D$ and $h\left(K_{i+1} / D \cap K_{i+1}\right)=1$. Since $K_{i+1}=$ is $_{K_{i}}\left(K\left(K_{i}\right)^{\prime}\right)$ we have $h(K / D \cap K)=1$; and since $K_{i}^{\prime} \subseteq D, D \triangleleft K_{i}$ and so $D \cap K \triangleleft K$. Since $K_{i+1}$ is isolated in $K_{i}$, the argument used in Step 1 to prove $W R K_{i+1}$ uniform also shows that $W R K_{i}$ is uniform. So for any nonzero submodule $W_{1}$ of $W, W_{1} R K_{i}$ is essential in $W R K_{i}$, and

$$
L_{i}=\left\{g \in L_{i-1}: W_{1} R K_{i} g \text { is } R K_{i} \text {-similar to } W_{1} R K_{i}\right\} \text {. }
$$

Hence, in proving $(1 ; i)$, we can replace $W$ by any of its nonzero $R K$-submodules. In particular, by Lemma 3.3, we may assume that

$$
u \text {-dim } \operatorname{din}_{R(D) K)}(W)<\infty \text {. }
$$

There exist $t \geqslant 0$ and elements $x_{0}, x_{1}, \ldots, x_{t}$ of $D$ such that, writing $D_{i}=$ $\left\langle K, x_{0}, \ldots, x_{i}\right\rangle$ for $0 \leqslant i \leqslant t, D_{0}=K, D_{t}=K D, D_{i} \triangleleft D_{i+1}$ and $D_{i+1} / D_{i}$ is infinite cyclic for $0 \leqslant i<t$. Clearly, $D \cap D_{i} \triangleleft D_{i+1}$. Hence, by hypothesis (iii) and $t$ applications of Lemma 3.4, starting from (3), $u$ - $\operatorname{dim}_{R D}(W R(K D))<\infty$. Since $E:=$ is $_{K_{i}}\left(K_{i+1} D\right)=$ is $_{K_{i}}(K D)$,

$$
u-\operatorname{dim}_{R D}(W R E)<\infty .
$$

By (iii), $W R K_{i}$ is a direct sum of conjugates of $W R E$ as an $R E$-module. Each of these summands has finite uniform dimension as an $R D$-module since $D \triangleleft K_{i}$. Thus every finitely generated $R E$-submodule of $W R K_{i}$ has finite uniform dimension as an $R D$-module.

Now let $g \in L_{i}$. Thus $W R K_{i}$ and $W R K_{i} g$ are similar uniform $R K_{i}$-modules. Let $V$ be a nonzero $R K_{i}$-module isomorphic to a submodule of both of them. Suppose that $K_{i+1} \neq K_{i+1}^{g}$ and put $E=$ is $_{K_{i}}\left(K_{i+1} K_{i+1}^{g}\right)$. There exists an isolated subgroup $D$ of $K_{i}$ with $K_{i+1}^{g} \subseteq D \triangleleft E$ and $h(E / D)=1$. Thus $D$ is of the type discussed in the previous paragraph. Note that is $K_{i}\left(K_{i+1} D\right)=E$. As shown above, every finitely generated $R E$-submodule of $W R K_{i}$, and hence of $V$, has finite uniform dimension as an $R D$-module.

But as an $R K_{i}$-module,

$$
W R K_{i} g=\left(W \otimes_{R K} R K_{i}\right) g \cong W g \otimes_{R K^{8}} R K_{i}=\left(W g \otimes_{R K^{g}} R D\right) \otimes R K_{i},
$$

where the isomorphism is given by $(w \otimes \alpha) g \mapsto w g \otimes \alpha^{g}$. Now $W g$ is a uniform $R K^{g}$-module, so the same is true of $W g \otimes_{R K^{g}} R D$ by Lemma 3.4. In view of (5), $V$, being isomorphic to a submodule of $W R K_{i} g$, contains an $R K_{i}$-submodule induced from $R D$, by Lemma 3.5; hence $V$ contains an $R E$-module $T$ induced from $R D$. In particular, $u$-dim $\operatorname{din}_{R D}(T)=\infty$, contradicting the analysis of the previous paragraph. Thus $K_{i+1}=K_{i+1}^{g},(1 ; i)$ is proved, and with it the theorem. 
5. Group theoretic lemmas. Recall that a locally nilpotent group is a $\mathscr{P}$-group if it is torsion free and all its finite rank subgroups are finitely generated. A subgroup $H$ of a group $G$ is dense in $G$ if is ${ }_{G}(H)=G$. The example of a free abelian group mapping onto $\mathbf{Q}^{+}$shows that $\mathscr{P}$ is not a quotient-closed class. Nevertheless, we do have

LEMMA 5.1. Let $G$ be a locally nilpotent group with torsion subgroup T. Suppose that $G / T$ is countable. Let $Z=Z(G)$.

(i) If $Z \in \mathscr{P}$, then the terms $Z_{r}(G) / Z_{r-1}(G)\left(r \in \mathbf{N}^{+}\right)$of the upper central series of $G$ are in $\mathscr{P}$. In particular, if $G$ is hypercentral of length $\omega$ and $Z \in \mathscr{P}$, then $G$ and $G / B$ are in $\mathscr{P}$, where $B=Z_{r}(G)$ for any $r \in \mathbf{N}^{+}$.

(ii) If $G$ is torsion free and in $\mathscr{P}$, then $G / Z \in \mathscr{P}$.

(iii) Let $B$ be a normal subgroup of $G$. Then there exists a dense subgroup $H$ of $G$ with $H$ and $H B / B$ in $\mathscr{P}$.

Proof. (i) If $Z \in \mathscr{P}$, the hypercentre of $G$ is torsion free, and so countable. The proof is by induction on $r$, the case $r=1$ being our hypothesis. Suppose $r>1$ and $Z_{r-1} / Z_{r-2}$ is in $\mathscr{P}$ (where $Z_{i}=Z_{i}(G)$ for all $i \in \mathbf{N}^{+}$). Let $Z_{r-1} \subseteq H \subseteq Z_{r}$, with $H / Z_{r-1}$ of finite rank. Let $x \in H \backslash Z_{r-1}$. There is an element $y$ of $G$ with $[x, y] \in Z_{r-1} \backslash Z_{r-2}$. The map $g \mapsto[g, y]$ induces a homomorphism $\theta: H / Z_{r-1} \rightarrow$ $Z_{r-1} / Z_{r-2}$ having nontrivial image of finite rank. By the induction hypothesis, this image is finitely generated and torsion free. The kernel of $\theta$ does not contain $Z_{r-1} x$. But $x$ was arbitrary, so $H / Z_{r-1}$ is residually-(finitely generated and torsion free). Hence there is a group $K$ with $Z_{r-1} \subseteq K \subseteq H$ and with $H / K$ finitely generated and torsion free of the same rank as $H / Z_{r-1}$. Since $H / Z_{r-1}$ is torsion free, $K=Z_{r-1}$. Hence $H$ is finitely generated.

(ii) Since $Z$ is torsion free, so is $G / Z$. This follows from the same result for nilpotent groups by an easy local argument. Let $H / Z$ be a finite rank subgroup of $G / Z$. We claim that there exists a subgroup $D$ of $G$, with $D / Z$ of finite rank, $H \subseteq D$ and $H \cap Z(D)=Z$. Suppose that such a group $D$ has been found. Then $D$ is nilpotent by [10, Theorem 6.36] applied to the torsion free, locally nilpotent, finite rank group $D / Z$. By Lemma 5.1(i), $D / Z(D) \in \mathscr{P}$. Since $H / Z \subseteq D / Z(D), H / Z$ is finitely generated. Thus an arbitrary finite rank subgroup of $G / Z$ is finitely generated; that is, $G / Z \in \mathscr{P}$.

To prove the claim, we define $D$ as follows. We shall choose $g_{1}, \ldots, g_{n} \in G$ such that $D=\left\langle H, g_{1}, \ldots, g_{n}\right\rangle$. Suppose $i \geqslant 1$ and $g_{1}, \ldots, g_{i-1}$ have been chosen, but $Z \subsetneq H \cap Z\left(\left\langle H, g_{1}, \ldots, g_{i-1}\right\rangle\right)$. Then choose $g_{i}$ to be any element of $G$ which does not centralise $H \cap Z\left(\left\langle H, g_{1}, \ldots, g_{i-1}\right\rangle\right)$. Thus

$$
H \cap Z\left(\left\langle H, g_{1}, \ldots, g_{i}\right\rangle\right) \varsubsetneqq H \cap Z\left(\left\langle H, g_{1}, \ldots, g_{i-1}\right\rangle\right),
$$

and the factor here is torsion free since factors of subgroups of $G$ by their centres always are, as observed at the start of the proof. Thus the process must stop since $H / Z$ has finite rank. Define $D$ to be the last group in the chain.

(iii) If $G=T$ take $H=\{1\}$. Otherwise, let $\left\{x_{i}: i \geqslant 1\right\}$ be a transversal to $T$ in $G$. The countable subgroup $U=\left\langle x_{i}: i \geqslant 1\right\rangle$ is dense in $G$, so we need only find a dense 
subgroup $H$ of $U$ with $H$ and $H / H \cap B$ both in $\mathscr{P}$. Since

$$
H / H \cap B=H / H \cap(U \cap B) \cong H(U \cap B) / U \cap B,
$$

we may assume that $G=U=\left\langle x_{i}: i \geqslant 1\right\rangle$. Put $K_{0}=1$ and for $i \geqslant 1$ put $K_{i}=$ $\left\langle x_{1}, \ldots, x_{i}\right\rangle$. Thus $K_{i}$ is nilpotent, so that $K_{i-1}$ is subnormal in $K_{i}$. By refining the series $K_{0} \subseteq K_{1} \subseteq K_{2} \subseteq \cdots$ we can therefore obtain a series $1=G_{0} \subseteq G_{1} \subseteq \cdots \subseteq$ $G=\bigcup_{i} G_{i}$ with $G_{i} / G_{i-1}$ cyclic for all $i \geqslant 1$.

Define dense subgroups $H_{i}$ of $G_{i}$ as follows. Set $H_{0}=1$ and suppose that $i \geqslant 1$ and $H_{i-1}$ has been defined. There are three cases to consider.

Case (i). $G_{i} / G_{i-1}$ finite. Put $H_{i}=H_{i-1}$.

Case(ii). $G_{i} / G_{i-1}$ and $G_{i} B / G_{i-1} B$ both infinite. Let $G_{i} / G_{i-1}=\left\langle G_{i-1} g\right\rangle$, so $G_{i} B / G_{i-1} B=\left\langle G_{i-1} B g\right\rangle$. A dense subgroup of a finitely generated nilpotent group is of finite index, so $H_{i-1}$ contains a characteristic subgroup $L_{i-1}$ of $G_{i-1}$ with $G_{i-1} / L_{i-1}$ finite. Now $g$ acts on $G_{i-1}$, and so on $G_{i-1} / L_{i-1}$. Thus some power, $g^{r}$ say, of $g$ fixes $G_{i-1} / L_{i-1}$ and in particular leaves $H_{i-1}$ invariant. Define $H_{i}=$ $\left\langle H_{i-1}, g^{r}\right\rangle$, the split extension of $H_{i-1}$ by $\left\langle g^{r}\right\rangle$, so $H_{i}$ is dense in $G_{i}$, and $H_{i} / H_{i-1}$ and $H_{i} B / H_{i-1} B$ are infinite cyclic.

Case(iii). $G_{i} / G_{i-1}=\left\langle G_{i-1} g\right\rangle$ infinite, and $G_{i} B / G_{i-1} B$ finite. Then $\left|G_{i} B: H_{i-1} B\right|$ $<\infty$, and we can choose $r \geqslant 1$ in a similar manner to Case (ii) so that $H_{i}:=\left\langle H_{i-1}, g^{r}\right\rangle$ is the split extension of $H_{i-1}$ by $\left\langle g^{r}\right\rangle$, and so that $g^{r} \in H_{i-1} B$. Thus $H_{i} / H_{i-1}$ is infinite cyclic, and $B H_{i}=B H_{i-1}$.

Each factor $H_{i} / H_{i-1}$ is either trivial or infinite cyclic. Put $H=\cup_{i} H_{i}$, so $H$ is torsion free and dense in $G$. A subgroup of $H$ having finite rank must lie in some $H_{i}$, and so be finitely generated. Similarly, $B H / B \in \mathscr{P}$. Thus $H$ is the required subgroup of $G$.

LEMMA 5.2. Let $G$ be a locally nilpotent group with $\langle g\rangle^{G}$ polycyclic for all $g \in G$. Let $B \triangleleft G$ be such that $G$ has the maximal condition on subgroups intersecting trivially with $B$. Then $G / B$ is of finite torsion free rank.

Proof. Let $A$ be an abelian subgroup of $G$ maximal such that $A \cap B=1$. Since $A$ has the maximal condition on subgroups, it is finitely generated. Thus $A^{G}$ is finitely generated. Let $C=C_{G}\left(A^{G}\right)$, so $G / C$ has the maximal condition on abelian subgroups, being a group of automorphisms of a polycyclic group [10, proof of Theorem 3.27]. Since $G / C$ is locally nilpotent, it is polycyclic by [10, Theorem 3.31].

Suppose that $G / B$ has infinite torsion free rank. Then $B C / B$ has infinite rank, so there exists $g \in C$ such that $B D / B$ has strictly greater rank than $B A / B$, where $D$ is the abelian group $\langle A, g\rangle$. Let $B_{0}=B \cap D$, so $\left|D: B_{0} \cap A\right|=\infty$. Hence there is a subgroup $E$ of $D$ with $A \varsubsetneqq E$ and $B_{0} \cap E=1$. This contradicts our choice of $A$. Therefore $G / B$ has finite rank.

6. Noetherian quotient rings. This section is devoted to the proof of Theorem B. The strategy employed here for proving that certain rings of fractions are Noetherian is embodied in the following lemma. 
LEMMA 6.1. Let $R$ be a ring which is the union of an ascending chain $\left\{S_{\lambda}: 0 \neq \lambda<\rho\right\}$ of right Noetherian subrings $S_{\lambda}$ (where $\rho$ is an ordinal). Assume that $R$ is a flat left $S_{\lambda}$-module for all $\lambda$. Then $R$ is right Noetherian if and only if, given $a$ nonzero $R$-module $M$, there exists $\lambda<\rho$ and a nonzero $S_{\lambda}$-submodule $N$ of $M$ with $N R=N \otimes_{S_{\lambda}} R$.

Proof. If $R$ is right Noetherian and $M$ is a nonzero $R$-module, then any nonzero cyclic submodule $M^{\prime}$ of $M$ has the form $R / I$ for a finitely generated right ideal $I$ of $R$. There exists $\lambda$ such that $I=\left(I \cap S_{\lambda}\right) R=\left(I \cap S_{\lambda}\right) \otimes_{S_{\lambda}} R$; so $M^{\prime}=N \otimes_{S_{\lambda}} R$, where $N=S_{\lambda} / I \cap S_{\lambda}$.

Suppose, on the other hand, that $R$ is not right Noetherian. Choose a right ideal $I$ of $R$ which is maximal with respect to not being finitely generated. (Such an ideal exists by Zorn's Lemma.) By hypothesis, there exist $\lambda$ and a nonzero cyclic $S_{\lambda}$-submodule $N$ of $R / I$ such that $N R=N \otimes_{S_{\lambda}} R$. Let $N R=K / I$, so, by choice of $I, K$ is finitely generated. By taking a greater $\lambda$ if necessary, we may assume that $K=K^{\prime} R=K^{\prime} \otimes_{S_{\lambda}} R$ for some right ideal $K^{\prime}$ of $S_{\lambda}$. There exists a right ideal $I^{\prime}$ of $S_{\lambda}$ with $I^{\prime} \subseteq K^{\prime}$ and $K^{\prime} / I^{\prime}=N$. Notice that in fact $I^{\prime}=I \cap K^{\prime}$. Since $R$ is a flat left $S_{\lambda}$-module, $K / I=\left(K^{\prime} / I^{\prime}\right) R=K^{\prime} \otimes R / I^{\prime} \otimes R$, and so $I=I^{\prime} \otimes R$ is finitely generated. This is a contradiction, so $R$ is right Noetherian.

Let $M$ be a finitely generated module over the Noetherian ring $S$. A critical composition series (c.c.s.) of $M$ is a series $0=M_{0} \subseteq M_{1} \subseteq \cdots \subseteq M_{n}=M$ of submodules of $M$ defined as follows. Set $M_{0}=0$, and if $M_{i}$ has been defined, let $M_{i+1}$ be a maximal member of the set of submodules $X$ of $M$ with $M_{i} \subset X$ and $X / M_{i}$ critical of smallest possible Krull dimension. Associated with each c.c.s. of $M$ is a sequence of nonnegative integers $\left(a_{0}, a_{1}, \ldots, a_{i}, \ldots\right)$, where $a_{i}$ indicates that there are $a_{i}$ factors in the c.c.s. of $M$ of Krull dimension $i$. (Note that these $i$ factors all occur together-the sequence of Krull dimensions of the factors of a c.c.s. is nondecreasing.) The sequence $\left(a_{1}, a_{2}, \ldots\right)$ is called the critical sequence of $M$; it follows from the analogue of the Jordan-Hölder theorem for c.c.s. [7, Corollary 2.8] that the critical sequence is an invariant of $M$. Critical sequences can be ordered lexicographically; this done, it is easy to see that the critical sequence of any proper factor of $M$ is strictly less than that of $M$. We shall need these ideas to prove

THEOREM B. Let $R$ be a commutative domain and let $G$ be a torsion free locally nilpotent hyperabelian $\mathscr{P}$-group. Let $B \triangleleft G$ with $G / B \in \mathscr{P}$, and let $Q G$ denote $R G(R B)^{-1}$. Then the following are equivalent:

(a) $Q G$ is Noetherian;

(b) the set of subgroups of $G$ having trivial intersection with $B$ satisfies the ascending chain condition;

(c) $G$ has no abelian subgroup $A$ of infinite rank with $B \cap A=1$.

Proof. Replacing $R$ by its quotient field, we may assume that $R$ is a field.

(a) $\Rightarrow$ (c) If a group $A$ exists as in (c), then $R A$ is not Noetherian. Since $Q G$ is a faithfully flat extension of $R A, Q G$ is not Noetherian. 
(c) $\Rightarrow$ (b) Suppose (c) holds but (b) fails to hold, and choose a subgroup $H$ of $G$ which is not finitely generated and satisfies $H \cap B=1$. Since $H \in \mathscr{P}, H$ is finitely generated nilpotent by (c) and [10, Theorem 6.36]. This is a contradiction.

(b) $\Rightarrow$ (a) We shall use Lemma 6.1.

Step 1. The induction set-up. There is a chain $B=B_{0} \subset \cdots \subset B_{\alpha}=G$ of normal subgroups of $G$ with $B_{\beta+1} / B_{\beta}$ abelian for all ordinals $\beta$ with $0 \leqslant \beta<\alpha$. This chain can be refined to a chain $B=G_{0} \subset \cdots \subset G_{\rho}=G$ such that $G_{\lambda} \triangleleft G_{\lambda+1}$ and $G_{\lambda+1} / G_{\lambda}$ is cyclic for $0 \leqslant \lambda<\rho$. The proof is by induction on the ordinal $\rho$, so by hypothesis

$$
R G_{\lambda}(R B)^{-1} \text { is Noetherian for all } \lambda<\rho .
$$

If $\rho=\lambda+1$, then $R G_{\rho}(R B)^{-1}$ is Noetherian by (1) and, if $\left|G_{\lambda+1}: G_{\lambda}\right|=\infty$, a twisted version of the Hilbert basis theorem [9, Theorem 10.2.6]. So we may assume that $\rho$ is a limit ordinal. Thus, by construction of the chain of $G_{\lambda}$ 's, $G$ is the ascending union of those $G_{\lambda}, \lambda<\rho$, which are normal in $G$.

Let $M$ be a nonzero $Q G$-module. We shall construct, in Step 3, sequences $1=H_{0} \subseteq H_{1} \subseteq \cdots$ and $G=L_{0} \supseteq L_{1} \supseteq \cdots$ of subgroups of $G$ with $H_{i}$ finitely generated, $H_{i} \triangleleft L_{i}$ for all $i \geqslant 0$, and $B H_{i} \subsetneq B H_{i+1}$ unless $L_{i+1}=H_{i+1}$; and elements $m_{i} \in M$ such that for $i \geqslant 0$,

$$
\begin{gathered}
m_{i} R G=m_{i} R L_{i} \otimes R G ; \\
m_{i} R H_{i} \text { is critical and impervious; }
\end{gathered}
$$

$m_{i} R H_{i}\left(L_{i} \cap B\right)=m_{i} R H_{i} \otimes R H_{i}\left(L_{i} \cap B\right)$, and there exists no $R H_{i}\left(L_{i} \cap B\right)$-submodule of $m_{i} R L_{i}$ isomorphic to a submodule of a proper quotient of $m_{i} R H_{i}\left(L_{i} \cap B\right)$;

and

(5;i) each uniform $R H_{i}$-submodule of $m_{i} R L_{i}$ is similar to $m_{i} R H_{i}$.

Step 2. Deduction of the theorem from the construction. Let $H=\cup_{i} H_{i}$ and $L=\bigcap_{i} L_{i}$, so $H$ is a normal subgroup of $L$. For each $i, H_{i} \triangleleft L$, so $H_{i} \triangleleft H$, and hence $H$ is a locally nilpotent group with $\langle h\rangle^{H}$ polycyclic for all $h \in H$. Since $G / B \in \mathscr{P}$, $B H / B$ is polycyclic by Lemma 5.2(ii). Hence there exists $t \geqslant 0$ such that $B H=B H_{t}$ $=B H_{t+i}$ for all $i \geqslant 0$. By construction, $L_{t+1}=H_{t+1}$, so that $B L_{t+1} / B$ is finitely generated. Therefore $R\left(B L_{t+1}\right)(R B)^{-1}$ is Neetherian and in view of $(2 ; t+1)$ we have achieved the conditions specified by Lemma 6.1. Thus $Q G$ is Noetherian.

Step 3. The construction of $H_{i}, L_{i}$ and $m_{i}$. Put $H_{0}=1, L_{0}=G$ and let $m_{0}$ be an arbitrary nonzero element of $M$. Let $i \geqslant 0$ and suppose $H_{i}, L_{i}$ and $m_{i}$ have been chosen satisfying the stated conditions. Now either (a) $m_{i} R L_{i}=m_{i} R H_{i} \otimes R L_{i}$, or (b) $m_{i} R L_{i} \neq m_{i} R H_{i} \otimes R L_{i}$. In case (a), put $m_{i+1}=m_{i}$ and $L_{i+1}=H_{i}=H_{i+1}$, so completing the $(i+1)$ st step in the construction (and in fact all subsequent steps). Suppose (b) holds. Then $m_{i} R G \neq m_{i} R H_{i} \otimes R G$, so there exists some $\lambda=\lambda(i)<\rho$ such that $H_{i} \subseteq G_{\lambda}$ and $m_{i} R G_{\lambda} \neq m_{i} R H_{i} \otimes R G_{\lambda}$.

Since $Q G_{\lambda}$ is Noetherian, we can choose $m_{i+1} \in m_{i} R L_{i}$ such that the critical sequence of $m_{i+1} Q G_{\lambda}$ is minimal among all $m^{\prime} Q G_{\lambda}$ with $m^{\prime} \in m_{i} R L_{i}$. Hence there is no $m^{\prime}$ in $m_{i} R L_{i}$ with $m^{\prime} Q G_{\lambda}$ isomorphic to a nonzero submodule of a proper 
quotient of $m_{i+1} R G_{\lambda}$. From $(2 ; i), m^{\prime} R G=m^{\prime} R L_{i} \otimes R G$ for any $m^{\prime} \in m_{i} R L_{i}$, and therefore $m^{\prime} R G_{\lambda}=m^{\prime} R\left(G_{\lambda} \cap L_{i}\right) \otimes R G_{\lambda}$. Hence,

$$
m_{i+1} R G_{\lambda}=m_{i+1} R\left(G_{\lambda} \cap L_{i}\right) \otimes R G_{\lambda}
$$

and

no nonzero $R\left(G_{\lambda} \cap L_{i}\right)$-submodule of $m_{i} R L_{i}$ is isomorphic to a submodule of a proper quotient of $m_{i+1} R\left(G_{\lambda} \cap L_{i}\right)$.

Since $Q G_{\lambda}$ is Noetherian, $m_{i+1} Q G_{\lambda}=Q G_{\lambda} / I$ for some finitely generated right ideal $I$ of $Q G_{\lambda}$. Let $H_{i+1}$ be a finitely generated subgroup of $G$ such that $R H_{i+1}$ contains a set of generators for $I$. It is easy to check that $I \cap R G_{\lambda}=$ $\left(I \cap R H_{i+1}\right) R G_{\lambda}$, so that

$$
m_{i+1} R G_{\lambda}=m_{i+1} R H_{i+1} \otimes_{R H_{i+1}} R G_{\lambda} .
$$

In fact, in view of (6), $H_{i+1}$ may be made smaller, if necessary, so that (8) still holds and $H_{i+1}$ is a finitely generated subgroup of $L_{i} \cap G_{\lambda}$. Also, choosing a new $m_{i+1}$ inside the original $m_{i+1} R H_{i+1}$, and a yet smaller $H_{i+1}$ if necessary, we may suppose that $m_{i+1} R H_{i+1}$ is impervious and critical. This is $(3 ; i+1)$. Because we are considering a submodule of the original $m_{i+1} R\left(L_{i} \cap G_{\lambda}\right)$, this new choice of $m_{i+1}$ still satisfies (6) and (7).

We now define $L_{i+1}$ and establish $(2 ; i+1),(4 ; i+1)$ and $(5 ; i+1)$.

Let $C$ be an arbitrary finitely generated subgroup of $L_{i}$ with $H_{i+1} \subseteq C$. We wish to apply Theorem A with $H_{i+1}=K, C \cap G_{\lambda}=H$ and $C=G$, and with $m_{i+1} R H_{i+1}$ $=W$ and $m_{i+1} R C=M$. All the hypotheses other than (ii) are evidently satisfied. We must show that

$$
\begin{aligned}
& \text { there exists no nonzero } R\left(C \cap G_{\lambda}\right) \text {-submodule of } m_{i+1} R C \\
& \text { isomorphic to a submodule of a proper quotient of } m_{i+1} R\left(C \cap G_{\lambda}\right) \text {. }
\end{aligned}
$$

Suppose (9) is false; let $U \subseteq m_{i+1} R C$ be a nonzero $R\left(C \cap G_{\lambda}\right)$-module with $U$ isomorphic to a submodule $U^{\prime} / U_{1}$ of $m_{i+1} R\left(C \cap G_{\lambda}\right) / U_{1}$ with $U_{1} \neq 0$. Then the $R\left(L_{i} \cap G_{\lambda}\right)$-submodule $U R\left(L_{i} \cap G_{\lambda}\right)$ of $m_{i+1} R\left(L_{i} \cap G_{\lambda}\right)$ would be isomorphic to some quotient of $\left(U^{\prime} / U_{1}\right) \otimes R\left(L_{i} \cap G_{\lambda}\right)$. But $C$ contains $H_{i+1}$ and so

$$
m_{i+1} R\left(C \cap G_{\lambda}\right)=m_{i+1} R H_{i+1} \otimes R\left(C \cap G_{\lambda}\right)
$$

by (8). Thus

$$
\left(m_{i+1} R\left(C \cap G_{\lambda}\right) / U_{1}\right) \otimes R\left(L_{i} \cap G_{\lambda}\right)
$$

is isomorphic to a proper quotient of $m_{i+1} R H_{i+1} \otimes R\left(L_{i} \cap G_{\lambda}\right)$. But, by (8), the latter is isomorphic to $m_{i+1} R\left(L_{i} \cap G_{\lambda}\right)$. Hence $U R\left(L_{i} \cap G_{\lambda}\right)$ is isomorphic to a submodule of a proper quotient of $m_{i+1} R\left(L_{i} \cap G_{\lambda}\right)$. This contradicts (7). Hence (9) holds.

Applying Theorem A, we deduce that $m_{i+1} R C=m_{i+1} R E \otimes_{R E} R C$, where $E=$ $N_{C}\left(H_{i+1}\right)$. Since $C$ was arbitrary in $L_{i}$ it follows that

$$
m_{i+1} R L_{i}=m_{i+1} R Y_{i+1} \otimes R L_{i},
$$

where $Y_{i+1}=N_{L_{i}}\left(H_{i+1}\right)$. 
Now set $L_{i+1}=\left\{g \in Y_{i+1}: m_{i+1} R H_{i+1}\right.$ is similar to $\left.m_{i+1} R H_{i+1} g\right\}$. By Lemma 3.2(i), $L_{i+1}$ is a group. Clearly, $H_{i+1} \triangleleft L_{i+1} \subseteq L_{i}$. By (7), no $R\left(L_{i} \cap G_{\lambda}\right)$-submodule of $m_{i+1} R Y_{i+1}$ is isomorphic to a submodule of a proper quotient of $m_{i+1} R\left(L_{i} \cap G_{\lambda}\right)$. Just as was argued in proving (9), one may deduce from this, using (8), that

$$
\begin{aligned}
& \text { no nonzero } R H_{i+1} \text {-submodule of } m_{i+1} R Y_{i+1} \text { is isomorphic to a } \\
& \text { submodule of a proper quotient of } m_{i+1} R H_{i+1} \text {. }
\end{aligned}
$$

From (11) and Lemma 3.1 follows $(6 ; i+1)$.

Now apply Lemma 3.2(ii) with $L_{i+1}=L, H_{i+1}=H$ and $Y_{i+1}=G$, and with $m_{i+1} R H_{i+1}=W$. In view of (11), we obtain

$$
m_{i+1} R Y_{i+1}=m_{i+1} R L_{i+1} \otimes R Y_{i+1} \text {. }
$$

Recalling that $m_{i+1} \in m_{i} R L_{i}$, we now deduce ( $\left.2 ; i+1\right)$ from (12), (10) and (2;i).

It remains to prove $(4 ; i+1)$ and that $H_{i} \subseteq H_{i+1}$ and $H_{i+1} \nsubseteq B H_{i}$.

First we prove $(4 ; i+1)$. Since $H_{i+1}\left(L_{i+1} \cap B\right) \subseteq G_{\lambda}$, (8) implies that

$$
m_{i+1} R H_{i+1}\left(L_{i+1} \cap B\right)=m_{i+1} R H_{i+1} \otimes R H_{i+1}\left(L_{i+1} \cap B\right) .
$$

The by now familiar argument now leads from (7) and (8) to the remainder of $(4 ; i+1)$. Hence $(4 ; i+1)$ is established.

Suppose next that $H_{i} \nsubseteq H_{i+1}$. Then $H_{i} \cap H_{i+1}$ is an isolated proper subgroup of $H_{i}$. So there is a normal isolated subgroup $H$ of $H_{i}$ satisfying $H_{i} \cap H_{i+1} \subseteq H \triangleleft H_{i}$. Since $G_{\lambda}$ contains $H_{i}$, (8) shows that $m_{i+1} R H_{i}=m_{i+1} R H \otimes R H_{i}$. Let $W$ be a uniform $R H$-submodule of $m_{i+1} R H$. Now $(5 ; i)$ says that there is a nonzero submodule $W_{1}$ of $W \otimes R H_{i}$ isomorphic to a submodule of $m_{i} R H_{i}$, and hence impervious. On the other hand, by Lemma 3.5 applied to $W \otimes R H_{i}, W_{1}$ contains a nonzero submodule induced from $R H$. This is a contradiction, and so $H_{i} \subseteq H_{i+1}$.

We prove next that

$$
H_{i} \subset H_{i+1} .
$$

We consider $m_{i} R G$, which as an $R G_{\lambda}$-module is a quotient of a sum of conjugates of $m_{i} R G_{\lambda}$. Suppose (13) is false, so that $H_{i}=H_{i+1}$. Then, by (8),

$$
m_{i+1} R G_{\lambda}=m_{i+1} R H_{i} \otimes R G_{\lambda} \text {. }
$$

Since $m_{i} R G \neq m_{i} R H_{i} \otimes R G_{\lambda}$, there is some finitely generated subgroup $D$ of $L_{i}$, containing $H_{i}$, for which $m_{i+1} R D \subseteq m_{i} R D$ and

$$
m_{i} R\left(D \cap G_{\lambda}\right) \nRightarrow m_{i} R H_{i} \otimes R\left(D \cap G_{\lambda}\right) .
$$

But $m_{i+1} R\left(D \cap G_{\lambda}\right)=m_{i+1} R H_{i} \otimes R\left(D \cap G_{\lambda}\right)$, and this module is a submodule of $\sum_{d \in D} m_{i} R\left(D \cap G_{\lambda}\right) d$. It therefore has a nonzero submodule isomorphic to a submodule of a quotient of $m_{i} R\left(D \cap G_{\lambda}\right) d$ for some $d \in D$. With (14) we can deduce that

$$
\begin{aligned}
& m_{i+1} R\left(D \cap G_{\lambda}\right) \text { has a nonzero submodule isomorphic to a } \\
& \text { proper quotient of }\left(m_{i} R H_{i} d\right) \otimes R\left(D \cap G_{\lambda}\right) .
\end{aligned}
$$

Let $W_{1}=m_{i+1} R H_{i}$ and $W_{2}=m_{i} R H_{i}$. By $(5 ; i)$ there is a nonzero $R H_{i}$-submodule $W_{1}$ isomorphic to a submodule of $W_{2}$. So $W_{1}$ and $W_{2}$ are critical modules of the same Krull dimension. So no nonzero submodule of $W_{1} d_{1}\left(d_{1} \in D \cap G_{\lambda}\right)$ can be 
isomorphic to a submodule of a proper quotient of $W_{2} d(d \in D)$. It follows from Lemma 3.6 that no nonzero submodule of $W_{1} \otimes R\left(D \cap G_{\lambda}\right)$ can be embedded in a proper quotient of $W_{2} d \otimes R\left(D \cap G_{\lambda}\right)$ for any $d \in D$. This contradicts (15) and thereby (13) is proved. This completes the proof of the theorem.

7. Primitive group rings. For the proof of Theorem $\mathrm{C}$ we need a variant of one of Zalesskii's intersection theorems. Let $G$ be a hyperabelian torsion free locally nilpotent group, so $G$ contains a series $1=G_{0} \subset G_{1} \subset \cdots \subset G_{\lambda} \subset \cdots \subseteq \cup_{\lambda} G_{\lambda}=$ $G$, where each $G_{\lambda}$ is normal in $G$, and $G_{\lambda+1} / G_{\lambda}$ is abelian for all ordinals $\lambda$. Define a characteristic subgroup $E(G)$ by induction on $\lambda$ as follows: (i) Put $E(G)=G$ if $G$ is abelian; (ii) if $\rho$ is a limit ordinal, put $E\left(G_{\rho}\right)=\cup_{\lambda<\rho} E\left(G_{\lambda}\right)$; (iii) put $E\left(G_{\lambda+1}\right)=$ $E\left(G_{\lambda}\right) C_{G_{\lambda+1}}\left(E\left(G_{\lambda}\right)\right)$. It is clear that $E(G)$ is a characteristic hypercentral subgroup of $G$, and that

$$
C_{G}(E(G)) \subseteq E(G) .
$$

Let a group $H$ act on a group $U$; we write

$$
\Delta_{H}(U)=\left\{u \in U:\left|H: C_{H}(u)\right|<\infty\right\} .
$$

If $x$ and $y$ are elements of a torsion free nilpotent group, and there exists $n \geqslant 1$ such that $\left[x, y^{n}\right]=1$, then $[x, y]=1$. Hence

$$
C_{G}(E(G))=\Delta_{E(G)}(G)
$$

and so by (1) and (2)

$$
\Delta_{E(G)}(G) \subseteq E(G)
$$

Put $3(G)=\Delta_{E(G)}(G)$, the Zalesskii subgroup of $G$. Note that $3(G)$ is the centre of $E(G)$. Applying [9, Theorem 8.4.10] with $H=E(G)$ and $W=3(G)$, we deduce part (i) of

Proposition 7.1. (i) Let $G$ be a torsion free hyperabelian locally nilpotent group with Zalesskii subgroup $3(G)$. If $k$ is a field and $I$ is a nonzero ideal of $k G$, then $I \cap k 3(G) \neq 0$.

(ii) If $G$ is torsion free and hypercentral, then the same conclusion holds as in (i), with $3(G)$ in this case taken to be the centre of $G$.

For Proposition 7.1(ii), see [9, Theorem 8.4.9].

The connection between Theorems $\mathrm{B}$ and $\mathrm{C}$ is afforded by the following special case of the results of [2].

LEMMA 7.2. Let $Z$ be a torsion free central subgroup of the group $H$, with $H / Z$ polycyclic. Let $k$ be a field and let $V$ be an irreducible $k H$-module. Then $V$ is not a faithful kZ-module.

Proof. By [2, Theorem 2.1], there exists $0 \neq \alpha \in k Z$ such that $V$ is $\alpha$-free; that is, there is a free $k Z$-submodule $M$ of $V$ with $V / M \alpha$-torsion. However, $V$ is irreducible and so, if $\operatorname{Ann}_{k Z}(V)=0, V$ is $k Z$-divisible. And, as first observed by P. Hall [8, Lemma 4.1], a nonzero $k Z$-module cannot be both $\alpha$-free and divisible. 
LEMMA 7.3. Let $G$ be a torsion free locally nilpotent group with centre $Z$. Let $k$ be a field and let $A$ be an abelian intersection subgroup for $k G$ (so that $Z \subseteq A$ ). Suppose that either $\operatorname{rank}(A / Z) \geqslant|k Z|$, or that $Z=1$. If $k$ is an algebraic extension of a finite field, assume that $A / Z$ has infinite rank. Then $k G$ is primitive.

Proof. By [1, Lemma 2], the hypotheses on $k, A$ and $Z$ allow one to find a maximal ideal $I$ of $k A$ with

$$
\{a \in A: a-1 \in I\}=1
$$

and

$$
I \cap k Z=0 .
$$

Put $J=\bigcap_{g \in G} I^{g}$. We shall show that

$$
J=0 \text {. }
$$

Let $H$ be a finitely generated subgroup of $A$, let $x \in G$ and let $\bar{H}$ be the normal closure of $H$ in $\langle H, x\rangle$. Since $G$ is locally nilpotent, $\bar{H}$ is a finitely generated subgroup of $A$. Hence $J \cap k \bar{H}=P_{1} \cap \cdots \cap P_{n}$, a finite intersection of prime ideals, each of which lies in $I^{g_{i}} \cap k \bar{H}$ for some $g_{i} \in G$. But $\left(I^{g_{i}}\right)^{+}=1$ from (3). Hence $P_{i}^{+}=1$ for each $i$, so

$$
P_{i}=\left(P_{i} \cap k C_{\bar{H}}(x)\right) k \bar{H}
$$

for each $i$, by [9, Corollary 11.4.6]. Therefore

$$
J \cap k \bar{H}=\left(J \cap k C_{\bar{H}}(x)\right) k \bar{H} .
$$

Now allow $H$ to range over all the finitely generated subgroups of $A$. From the resulting equalities (6), we deduce that $J=\left(J \cap k C_{A}(x)\right) k A$. And these equalities, as $x$ ranges over $G$, yield

$$
J=(J \cap k Z) k A .
$$

Now (5) follows from (4) and (7).

Let $X$ be a maximal right ideal of $k G$ containing $I G$, and let $V=k G / X$ have annihilator $P$. Then $P \cap k A \subseteq I$ and $P \cap k A$ is $G$-invariant, so $P \cap k A=0$ by (5). Since $A$ is an intersection subgroup, $P=0$; that is, $V$ is faithful.

THEOREM C. Let $G$ be a countable torsion free hyperabelian locally nilpotent group and let $k$ be a field. Let $Z=Z(G)$.

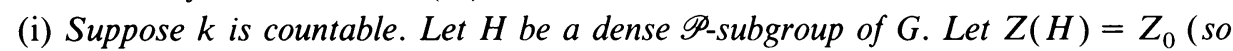
$\left.Z_{0}=Z \cap H\right)$. Consider the statements

(a) $k G$ is primitive;

(b) there exists an abelian subgroup $A$ of $G$, of infinite rank, with $Z \cap A=1$;

(c) $k H\left(k Z_{0}\right)^{-1}$ is not Noetherian.

Then (c) $\Leftrightarrow$ (b) $\Rightarrow$ (a). If $G$ and $G / Z$ are in $\mathscr{P}$, then (a) $\Rightarrow$ (c).

(ii) Suppose $k$ is uncountable. Then $k G$ is primitive if and only if $Z=1$.

Proof. Note that $H$ exists, by Lemma 5.1.

(i) (c) $\Leftrightarrow$ (b) Theorem B and Lemma 5.1(ii). 
(b) $\Rightarrow$ (a) Let $§(G)$ be the Zalesskii subgroup constructed before Proposition 7.1, so $Z \subseteq 3(G)$. If $3(G) / Z$ has infinite rank, then $k G$ is primitive by Lemma 7.3 with $A=3(G)$.

Suppose that $W:=3(G) / Z$ has finite rank. By [10, Lemma 6.37] applied to the (locally nilpotent) group $3(G) A / Z, A / C_{A}(W)$ has finite rank. We can thus assume that $A$ centralises $W$, so that

$$
3(G) A \text { is nilpotent. }
$$

Put

$$
Z_{1}=\{x \in 3(G):[x, A]=1\}=\Delta(3(G) A) \cap 3(G) .
$$

Replacing $A$ by a proper subgroup of infinite rank if necessary, we can assume that $Z_{1} \cap A=\left[Z_{1}, A\right]=1$. As in [1, Lemma 2], construct a maximal ideal $X$ of $k\left(Z_{1} A\right)$ with $X \cap k Z_{1}=0$ and $X^{+}=1$. Let $Y$ be a maximal right ideal of $k G$ with $X k G \subseteq Y$, and set $V=k G / Y$. Let $P=\operatorname{Ann}(V)$. Suppose that $P \neq 0$. Then $J:=P \cap k 3(G)$ $\neq 0$. If $J^{+} \neq 1$, then $B:=(J k(3(G) A))^{+} \neq 1$ and so $B \subseteq 3(G)$ and $B \cap$ $Z(3(G) A) \neq 1$ by $(8)$. Thus

$$
J^{+} \cap Z_{1} \neq 1
$$

But

$$
J \cap k Z_{1} \subseteq X \cap k Z_{1}=0,
$$

so (9) is impossible. Therefore $J^{+}=1$. The argument used in the proof of Lemma 7.3 can now be imitated to show that $J=\left(J \cap k Z_{1}\right) k 8(G)$. By $(10), J=0$. This is a contradiction, so $P=0$ and $V$ is a faithful irreducible $k G$-module.

Suppose now that $G$ (and so $G / Z$ ) are in $\mathscr{P}$. By the equivalence of (b) and (c), to prove that (a) implies (c) it is enough to show that

$$
\text { if } k G \text { is primitive, then } k G(k Z)^{-1} \text { is not Noetherian. }
$$

Suppose that $k G$ is primitive with a faithful irreducible module $V=k G / Y$. Write $Q G$ for $k G(k Z)^{-1}$ and assume that $Q G$ is Noetherian. Then $Y Q G$ is a finitely generated right ideal, so there exists a subgroup $D$ of $G$ with $Z \subseteq D$ and $D / Z$ finitely generated such that

$$
Y Q G=(Y \cap k D) Q G
$$

We claim that

$$
Y=(Y \cap k D) k G .
$$

Let $y \in Y$. By (12) there exists $0 \neq \alpha \in k Z$ such that $Y=\sum_{i} y_{i} g_{i} \alpha^{-1}$, where $y_{i} \in$ $Y \cap k D$ and $\left\{g_{i}\right\}$ is part of a right transversal to $D$ in $G$. Write $y=\Sigma_{i} \beta_{i} g_{i}$, with $\beta_{i} \in k D$. Therefore $\sum_{i} \beta_{i} \alpha g_{i}=\sum_{i} y_{i} g_{i}$, and so $\beta_{i} \alpha=y_{i} \in Y \cap k D$ for all $i$. Since $Y \cap k Z=0, k Z$ acts torsion freely on $V$, and so $\beta_{i} \in Y$ for all $i$. Thus $y \in$ $(Y \cap k D) k G$, and (13) is proved.

Put $M=k D / Y \cap k D$, so $V=M \otimes_{k D} k G$ by (13), and $M$ is irreducible. By Lemma 7.2, $0 \neq I=\operatorname{Ann}_{k Z}(M)$. But $V I=0$, and so $V$ is not faithful. This is a contradiction, so (11) is proved. 
(ii) If $k$ is uncountable and $k G$ is primitive, $Z=1$ by [9, Theorem 9.1.6]. Suppose now that $k$ is uncountable and $Z=1$. Then $G$ contains an abelian subgroup of infinite rank by [10, Theorem 6.36]. Let $F$ be the prime subfield of $k$. Now $F G$ is primitive by (b) $\Rightarrow$ (a) of part (i). Since $\Delta(G)=Z=1, k G$ is primitive by [9, Theorem 9.1.5].

Notes. (i) In fact, (a) $\Rightarrow$ (b) of Theorem C(i) remains true even if $G \notin \mathscr{P}$. For, a result of Brookes and Heineken (to appear) states that if a group $G$ satisfying the hypotheses of Theorem $\mathrm{C}$ has no subgroups $A$ as in (b), then $G$ is nilpotent; so one can prove (a) $\Rightarrow$ (b) by appealing to Theorem $\mathrm{D}$.

(ii) The only reason for requiring $G$ to be countable in Theorem C(i) is to be sure of the existence of a dense $\mathscr{P}$-subgroup $H$. We do not know whether such an $H$ always exists.

LEMMA 7.4. Let $H$ be a dense subgroup of the torsion free locally nilpotent group $G$. Let $k$ be a field.

(i) If $k H$ is primitive, then $k G$ is primitive.

(ii) If $G$ is hypercentral and if $k G$ is primitive, then $k H$ is primitive.

Proof. (i) Let $X$ be a maximal right ideal of $k H$ containing no nonzero ideal. Let $X k G \subseteq Y, Y$ a maximal right ideal of $k G$, and let $P$ be the annihilator of $k G / Y$. The ring $k G(k H)^{-1}$ is locally Artinian and is therefore the quotient division ring of $k G$. Thus if $P \neq 0, P \cap k H$ is a nonzero ideal of $k H$ contained in $Y \cap k H=X$. Since this is impossible, $P=0$.

(ii) Let $Z$ be the centre of $H$, so $Z=Z(G) \cap H$ since $G$ is torsion free. Let $k G / Y$ be a faithful irreducible $k G$-module. Let $0 \neq f \in k Z$, so there exists $\gamma \in k G$ with

$$
f \gamma \equiv \gamma f \equiv 1 \quad(\bmod Y) \text {. }
$$

The argument used in the proof of [2, Theorem C] shows that $\gamma$ may be chosen in $k H$. Let $X$ be a maximal right ideal of $k H$ containing $Y \cap k H$. By (14) (with $\gamma \in k H), f \notin X$. Hence, $X \cap k Z=0$. By Proposition 7.1(ii), $k H / X$ is a faithful irreducible $k H$-module.

THEOREM D. Let $G$ be a countable hypercentral group with $G \neq 1$ and let $k$ be $a$

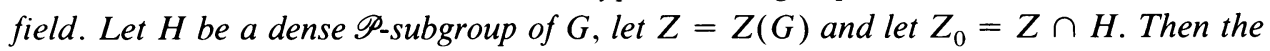
following are equivalent:

(a) $k G$ is primitive;

(b) $k$ is countable, $G$ is torsion free and there is an abelian subgroup $A$ of $G$, of infinite rank, with $A \cap Z=1$;

(c) $k H\left(k Z_{0}\right)^{-1}$ is not Noetherian, $G$ is torsion free and $k$ is countable.

Proor. (b) $\Rightarrow$ (a) This follows from Proposition 7.1(ii) and [3, Lemma 3.1].

(a) $\Rightarrow$ (c) If $k G$ is primitive, then it is prime, and so $G$ has no nonidentity finite normal subgroups. Thus $G$ is torsion free. By [9, Theorem 9.1.6], $k$ is countable. By Lemma 7.4(ii), $k H$ is primitive. Since $Z(H)=Z_{0}, H / Z_{0} \in \mathscr{P}$ by Lemma 5.1(i); and (a) $\Rightarrow$ (c) of Theorem C(i) shows that $k H\left(k Z_{0}\right)^{-1}$ is not Noetherian.

(c) $\Rightarrow$ (b) This follows from Theorem $\mathrm{C}(\mathrm{i})$. 


\section{REFERENCES}

1. C. J. B. Brookes, The primitivity of group rings of soluble groups with trivial periodic radical, J. London Math. Soc. (to appear).

2. K. A. Brown, The Nullstellensatz for certain group rings, J. London Math. Soc. (2) 26 (1982), 425-434.

3. _ Primitive group rings of soluble groups, Arch. Math. 36 (1981), 404-413.

4. W. D. Burgess, Rings of quotients of group rings, Canad. J. Math. 21 (1969). 865-875.

5. C. W. Curtis and I. Reiner, Methods of representation theory. Vol. I, Wiley-Interscience, New York, 1981.

6. R. Gordon and J. C. Robson, Krull dimension, Mem. Amer. Math. Soc. No. 133 (1973).

7. R. Gordon, Gabriel and Krull dimension, Ring Theory, Proc. Oklahoma Conf., Dekker, New York, 1974, pp. $241-295$.

8. P. Hall, On the finiteness of certain soluble groups, Proc. London Math. Soc. (3) 9 (1959), 595-622.

9. D. S. Passman, The algebraic structure of group rings, Wiley-Interscience, New York, 1977.

10. D. J. S. Robinson, Finiteness conditions and generalised soluble groups. Vols. I, II, Springer-Verlag, Berlin, 1972.

11. J. E. Roseblade, Prime ideals in group rings of polycyclic groups, Proc. London Math. Soc. 36 (1978), 385-447.

12. D. Segal, On the residual simplicity of certain modules, Proc. London Math. Soc. 34 (1977), 327-353.

13. R. C. Shock, Polynomial rings over finite dimensional rings, Pacific J. Math. 42 (1972), 251-257.

14. P. F. Smith, Quotient rings of group rings, J. London Math. Soc. (2) 3 (1971), 645-660.

Corpus Christi College, Cambridge CB2 1RH, United Kingdom

Department of Mathematics, University of Glasgow, University Gardens, Glasgow G12 8QW, UNITED KINGDOM 